\title{
Automatic Recognition of Multiple Affective States in Virtual Rehabilitation by Exploiting the Dependency Relationships
}

\author{
Jesús Joel Rivas \\ Computer Science Department \\ Instituto Nacional de Astrofísica, \\ Óptica y Electrónica (INAOE) \\ Puebla, México \\ jrivas@inaoep.mx \\ Amanda Williams \\ Clinical, Educational and Health Psychology \\ University College of London (UCL) \\ London, United Kingdom \\ amanda.williams@ucl.ac.uk
}

\author{
Felipe Orihuela-Espina \\ Computer Science Department \\ Instituto Nacional de Astrofísica, \\ Óptica y Electrónica (INAOE) \\ Puebla, México \\ f.orihuela-espina@inaoep.mx
}

\author{
Luis Enrique Sucar \\ Computer Science Department \\ Instituto Nacional de Astrofísica, \\ Óptica y Electrónica (INAOE) \\ Puebla, México \\ esucar@inaoep.mx
}

\author{
Nadia Bianchi-Berthouze \\ UCL Interaction Centre \\ University College of London (UCL) \\ London, United Kingdom \\ n.berthouze@ucl.ac.uk
}

\begin{abstract}
The automatic recognition of multiple affective states can be enhanced if the underpinning computational models explicitly consider the interactions between the states. This work proposes a computational model that incorporates the dependencies between four states (tiredness, anxiety, pain, and engagement) known to appear in virtual rehabilitation sessions of post-stroke patients, to improve the automatic recognition of the patients' states. A dataset of five stroke patients which includes their fingers' pressure (PRE), hand movements (MOV) and facial expressions (FAE) during ten sessions of virtual rehabilitation was used. Our computational proposal uses the Semi-Naive Bayesian classifier (SNBC) as base classifier in a multiresolution approach to create a multimodal model with the three sensors (PRE, MOV, and FAE) with late fusion using SNBC (FSNB classifier). There is a FSNB classifier for each state, and they are linked in a circular classifier chain (CCC) to exploit the dependency relationships between the states. Results of CCC are over $90 \%$ of ROC AUC for the four states. Relationships of mutual exclusion between engagement and all the other states and some co-occurrences between pain and anxiety for the five patients were detected. Virtual rehabilitation platforms that incorporate the automatic recognition of multiple patient's states could leverage intelligent and empathic interactions to promote adherence to rehabilitation exercises.
\end{abstract}

Index Terms-automatic affective states recognition, virtual rehabilitation, multi-label classification, classifier chains, stroke

This project was supported by a grant from the Secretaría de Educación, Ciencia, Tecnología e Innovación de la Ciudad de México. SECITI/042/2018 (INGER-DI-CRECITES-001-2018) "Red Colaborativa de Investigación Traslacional para el Envejecimiento Saludable de la Ciudad de México" (RECITES), and a grant from the project FORDECyT 296737 "Consorcio en Inteligencia Artificial". We would also like to acknowledge support for the Scholarship No. 434867 from the Mexican Research Council CONACYT.

\section{INTRODUCTION}

Virtual rehabilitation platforms provide opportunities to incorporate technologies for monitoring patients' performance and for controlling virtual scenarios that can leverage intelligent and empathic interactions with the patients, to promote adherence to the rehabilitation exercises [1]-[4]. When the platform is able to detect the affective, physical and/or psychological states of the patients, there are more opportunities for creating empathic and motivating interactions. The problem is that it is not easy to detect the affective states of interest with precision.

Some affective states are more likely to co-exist, while others do not; indeed others are mutually exclusive. For example, a piece of music can elicit mixed emotions of relaxed-calmsad, but it is improbable that it elicits the emotions of surprise and quietness, or relaxed and angry at the same time [5]. In the context of chronic pain rehabilitation, patients co-experience anxiety, and fear often expressed in the form of protective behaviour [6].

Given the existence of such co-occurrence and mutually exclusive relationships between affective states, they could be exploited to improve the discrimination between these states [7], [8]. Indeed, a fundamental factor that could contribute to the robustness of the automatic recognition of affective states is when computational models consider interactions of the affective states of interest.

Consequently, this work aims to propose a computational model that takes advantage of the dependency relationships between the affective, physical and/or psychological states involved in the rehabilitation of patients after stroke, to leverage the automatic recognition of the patients' states in a virtual 
rehabilitation platform. For this purpose, a dataset of 5 stroke patients was used. This dataset includes finger pressure (from PRE sensor), hand movements (from MOV sensor), and facial expressions (from FAE sensor) of the patients during ten rehabilitation sessions using a virtual rehabilitation platform called Gesture Therapy [1], [2]. The patients' states considered in this study were tiredness, anxiety, pain, and engagement.

Our proposal uses as base classifier a derivation from Naive Bayes classifier, named Semi-Naive Bayesian classifier (SNBC) [9], for its efficiency, simplicity and because it tackles dependent features [10]. SNBC is used to build the Multiresolution SNBC (MSNB) [11] and then to create the late Fusion of the three sensors (PRE, MOV, and FAE) using SNBC (FSNB). Finally, the states dependency relationships are exploited by a multi-label classifier named circular classifier chains (CCC) [12]. Particularly, the fusion of PRE, MOV, and FAE using FSNB classifier was studied in a previous work [13] whose main purpose was to investigate the contribution of each modality to the automatic recognition. The FAE modality achieved the highest recognition rates (over $90 \%$ in area under the curve (AUC) for all the states), and it seems that the psychiatrists labelling had much influence on these results because they paid special attention to the patients' face. For PRE and MOV, FSNB achieved over $82 \%$ in AUC, except for pain were the recognition rates were around $70 \%$ in AUC. Our Bayesian approach of MSNB classifier was compared against SVM and random forest [11], and the results were promising. An advantage of the Bayesian approaches is that their models are interpretable.

An experiment was carried out using the dataset of poststroke patients mentioned above. In this experiment, $\mathrm{CCC}$ performance was compared against the performance of the multi-label classifiers: Binary Relevance (BR) and Classifier Chains (CC). The three classifiers used FSNB as the base classifier. The conditional probability tables (CPTs) created automatically by CCC using FSNB classifier were analyzed for trying to determine which dependency relationships between the states were captured.

\section{RELATED WORK}

Some relationships of co-occurrence and mutual exclusion between emotions were exposed in studies to elicit emotions by watching video clips [14], [15]. The emotions of anger and disgust were difficult to induce independently [14]. Evidence suggested that one emotion could trigger another; for example, anger may induce anxiety [14]. Other videos clips that induce contentment, amusement, also elicit happiness, but it did not happen that the videos that induce anger could induce levels of happiness at the same time [15]. When music is used to generate emotions, some music pieces can induce mixed emotions of relaxed-calm-sad, but it is improbable that they elicit the emotions of surprise and quietness, or relaxed and angry at the same time [5]. In the health field, chronic pain patients exhibit protective behaviour during exercise in response to their anxiety, fear towards, and low confidence in such movements [16]. In addition, the work reported in
[6] shows how the relationship between pain and protective behaviour is mediated by anxiety.

Very few works consider dependence relationships between emotions and multidimensional classification [8]. The works of [17] and [8] exploit these characteristics. In [17], a Bayesian network is used to learn the relations of co-occurrence and mutual exclusion between pairs of emotions; but this is somewhat limiting because it does not include dependency relationships between more than two emotions simultaneously. In [8], a three-layer Boltzmann restrictive machine is used to identify the relations of dependence between more than one pair of emotions; but the problem is the computational cost involved in training and making inferences in a Boltzmann machine. In our proposal, we consider dependency relationships of two or more affective states by using circular classifier chains [12] where the predicted classes of the previous affective states in the chain are incorporated as additional feature inputs to the succeeding classifiers. Our core classifier is the SNBC which maintains the efficiency and simplicity of the Naive Bayesian classifiers [10].

\section{A Virtual Rehabilitation Platform: Gesture} THERAPY

Gesture Therapy (GT) [1], [2] is an upper limb virtual reality based rehabilitation platform for post-stroke patients. The platform is portable, thus patients can perform arm movements exercises at home or at the rehabilitation centre (Fig. 1).

GT integrates five interacting modules [2]: (a) Physical System (the hardware elements), composed of a personal computer, a webcam, and a hand grip (gripper) which is held by the patient's affected hand; (b) Tracking System, for tracking the gripper's colour ball and for detecting the finger pressure exerted on the pressure sensor (PRE sensor) in the gripper; (c) Simulated Environment, to display the serious games, and to control the interaction with the patient; (d) Trunk Compensation Detector, to indicate whether the patient makes compensation; and (e) Adaptation System, for adjusting the games' difficulty levels dynamically to obtain real-time customizability and adaptability to patient requirements and progress; here is where the recognition of affective states will be useful. GT also includes capabilities for recording performance using a video capture through the webcam. These recordings are helpful for extracting the facial expressions of the patient (FAcial Expression: FAE sensor). The tracker system estimates the 3D coordinates of hand movements (MOV sensor) and the finger pressure (PRE sensor) value at each frame, and sends this information to the simulated environment so that the patient interacts with the games and observes the actions in the computer screen.

\section{DATASET}

A dataset of post-stroke patients [11] was used to assess the performance of the proposed computational models which exploits the dependency relationships between the patient's affective states. This dataset contains the performance records 


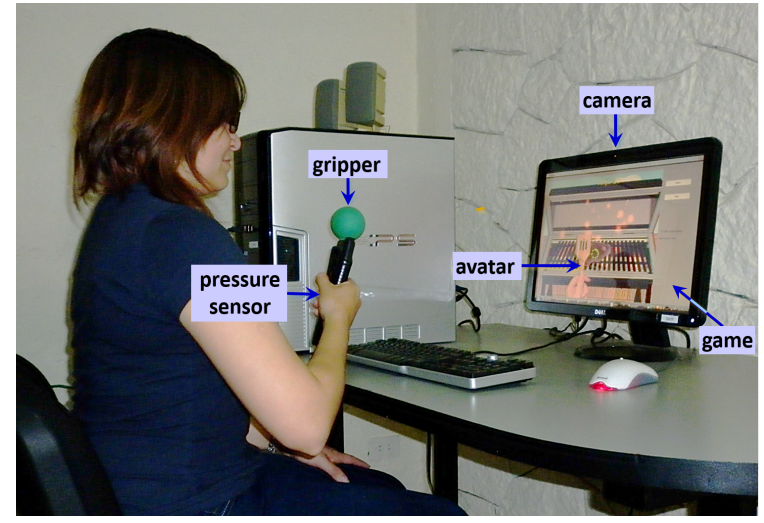

Fig. 1. A person using the Gesture Therapy platform. The person is holding the gripper with her right hand. The gripper has a frontal sensor for registering finger pressure. The webcam follows the gripper's colour ball to control an avatar (in this case is the hand with the kitchen palette on the screen) in the game (virtual environment).

of 5 post-stroke patients while using the GT system during ten sessions over a period of about one month (each session was taken in a different day, maximum 3 sessions per week). Patients played 5 games, each one with at most 3 minutes (therapist decided how much of that time should be employed by each patient on each game). Data collected consisted of frontal videos of patients (where spontaneous facial expressions, postures of the upper torso, and hand movements could be observed), a finger pressure value, and 3D hand position at each video frame. Data were labelled frame by frame by psychiatrists using four patients' states (affective, physical or psychological) representing tiredness, anxiety, pain, and engagement.

Feature vectors were created with a sliding window (of a predefined size) over consecutive frames of the respective data (see subsection V-B). There lead to a feature vector for each step forward of the sliding window. The feature vector for finger pressure (from PRE sensor) has 3 features (averages of the data contained in the sliding window): pressure (Pres), pressure speed (PresSpe) and pressure acceleration (PresAce). For hand movements, the feature vector (from MOV sensor) has 5 features (averages of the data contained in the sliding window): speed (Spe), acceleration (Ace) and differential location by the axes: $x$ (DifLx), $y$ (DifLy), $z$ (DifLz). Finally, for the facial expressions, 20 features from each frame of the patients' frontal video were extracted [18]. These features by frame represent distances or angles of geometrical figures over the eyebrows, the eyes and the mouth [19] (Fig. 2). Then, the feature vector (from FAE sensor) contains 20 averaged features (averages of the data contained in the sliding window) for $F 1$ $(a v F 1), F 2(a v F 2), \ldots, F 20(a v F 20)$. All the feature vectors have four binary tags (from the set $\{-1,1\}$ ), one for each state (tiredness, anxiety, pain, and engagement), indicating the presence (1) or the absence $(-1)$ of the state. Since the data were labelled frame by frame, the corresponding tag was generated as the majority label in the sliding window. Therefore, data of the three sensors and the classes tags were

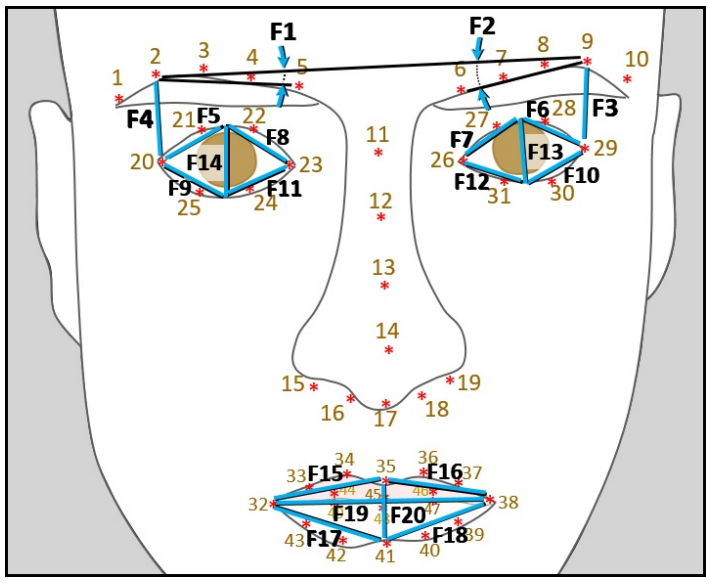

Fig. 2. Facial Expressions Features: $F_{i}, i \in\{1,2, \cdots, 20\}$. These features are distances or angles of geometrical figures over the eyebrows, the eyes, and the mouth at a video frame.

synchronized through the associated frames.

\section{Computational Models: SNBC, MSNB2, FSNB, AND CCC}

The following computational models were assembled to obtain the final model which includes the affective states' relationships to improve automatic recognition. The basic model was the SNBC which was the fundamental model to build all the other models. Then, for processing each sensor, i.e., each modality for affective states recognition, we used the Multiresolution Semi-Naive Bayesian (MSNB) classifier (with a modification, and we call the new model, MSNB2). The MSNB2 for each sensor estimated the presence or the absence of the same affective state and a late Fusion using SNBC (FSNB) was implemented finally to recognize the occurrence of the affective state. There were as many FSNB classifiers as affective states, each one for recognizing one affective state. These FSNB classifiers were linked in a circular classifier chain (CCC) which integrated the interactions of the affective states to enhance the final recognition.

\section{A. Semi-Naive Bayesian classifier (SNBC)}

Semi-Naive Bayesian classifier (SNBC) is based on Naive Bayes classifier (NBC) [9], [20]. Given a sample $s_{a}=\left(a_{1}, a_{2}, \cdots, a_{n}\right)$, and given the $i t h$ feature $A_{i}$, the decision rule of NBC for a two class problem (the class variable $C$ takes values in $\{-1,1\}$ ), is expressed as:

$\operatorname{class}\left(s_{a}\right)=\underset{c \in\{-1,1\}}{\arg \max }\left(\operatorname{Prob}(C=c) \prod_{i=1}^{n} \operatorname{Prob}\left(A_{i}=a_{i} \mid C=c\right)\right)$

The multiplication in (1) is supported by the naive assumption that all features $A_{i}$ are independent given the class $C$ [9]. To address a more generic and realistic situation, the SNBC executes a structural improvement [9], [21], [22] to remove and/or to join features (to eliminate redundant or 
irrelevant features and/or join dependent features). The structural improvement (Fig. 3) employs mutual information and conditional mutual information calculations [23] between the features and the class to make the improvements. After each operation of elimination or join of features, the new structure is tested to determine whether classification performance is improved. The process is repeated until all features have been analyzed.

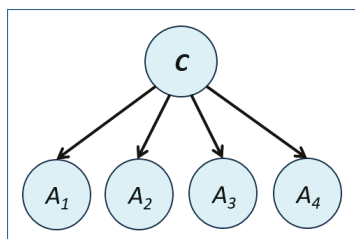

(a) Initial structure

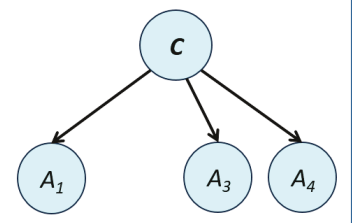

(b) Elimination of $A_{2}$

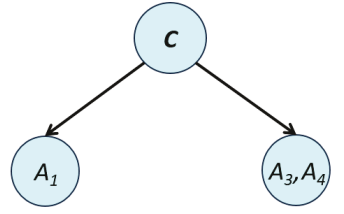

(c) Joining $A_{3}$ and $A_{4}$

Fig. 3. Example of the process of structural improvement to obtain a SemiNaive Bayesian (SNB) model: (a) An original Naive Bayes model with 4 features (all of them are assumed independent), (b) Feature $A_{2}$ is eliminated because the mutual information value between $A_{2}$ and the class $C$ is close to zero, (c) Features $A_{3}$ and $A_{4}$ are joined into one, as they are considered dependent base upon the conditional mutual information value between $A_{3}$ and $A_{4}$ given the class $C$.

\section{B. Multiresolution Semi-Naive Bayesian (MSNB2) classifier}

Multiresolution Semi-Naive Bayesian classifier (MSNB) is a binary classifier to explore the occurrence of an affective state of interest in the trace over time [24]. The classifier operationalizes several odd-size windows (starting from 3) concentric to a current frame. These windows are shifted simultaneously over the trace, to calculate several features in the environment of the current frame (neighbourhood). There is a SNBC associated with each window to discriminate the presence or not of the affective state in the corresponding window. (Fig. 4). The name multiresolution is used because the windows represent several concurrent resolutions at the current frame of the trace. Therefore, the associated SNBCs constitute simultaneous sliding estimators at different resolutions. MSNB represents an ensemble of SNBCs with a late (decision level) fusion process by majority vote. Each SNBC receives the features coming from a different window size and infers the presence or not of the affective state of interest. Finally, in the fusion stage, the presence or not is decided, by means of the majority vote of the SNBCs. Since the input features are numeric values, we employed a discretization process called Proportional k-interval discretization (PKID) [25].

A modification was made to MSNB classifier replacing the majority voting with a SNBC in the late fusion module, and the resulting classifier was called MSNB2. In part a) of Fig. 5 the architecture of MSNB2 is shown.

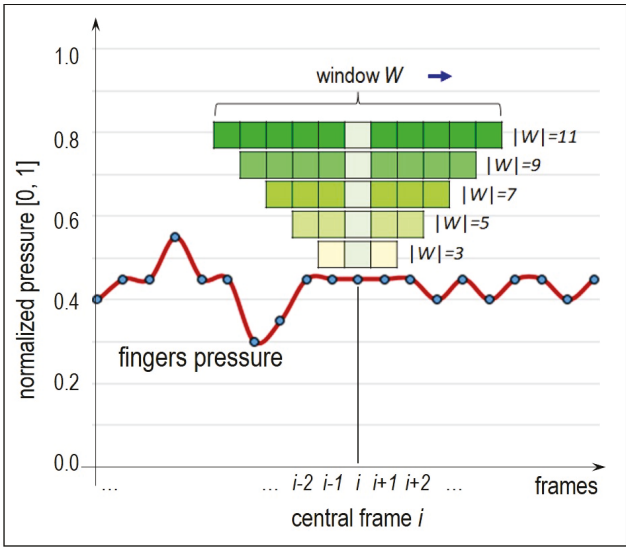

Fig. 4. Multiresolution process using several odd-size windows $(3,5,7,9$, and 11) concentric to a current frame $f_{i}$. Windows are shifted simultaneously over the trace. Exemplification corresponds to the trace of finger pressure at each video frame during a segment of a rehabilitation session. A SNBC is trained for each window size to infer the presence (1) or absence (-1) of the affective state into consideration. Then, each of the 5 SNB models (a model for each one of the 5 windows) returns its prediction for the class tag in each sample $f_{i}$ of the series, and the MSNB2 makes a late fusion using a SNBC to assign the final class tag ( 1 or -1$)$ to $f_{i}$.

\section{Late Fusion using SNBC (FSNB)}

There is a separate MSNB2 classifier for each sensor (PRE, $\mathrm{MOV}$, and FAE) to predict the occurrence of an affective state. Then, the predicted classes of the three MSNB2 are fused using a SNBC (FSNB) [13]. Therefore, FSNB is a binary classifier which represents a multimodal affective states recognizer (Fig. 5, part b)).

\section{Circular Classifier Chains (CCC)}

There are as many FSNB classifiers as affective states, each one for recognizing one state. These FSNBs classifiers are linked in a circular architecture, so they participate as the base classifiers in a multi-label classification process called Circular Classifier Chains (CCC), where the interactions of the affective states were considered.

CCC [12] is an extension to Classifier Chains (CC) [26] for addressing the problem of defining the class variables' ordering in the chain. CC is related to Binary Relevance (BR), an approach which consists of $q$ base binary classifiers for classifying $q$ class variables, where each one is independently trained to predict the occurrence of a class variable. CC incorporates class interactions to the BR approach through a strategy of creating a chain where each classifier includes as additional features the predicted classes of the previous classifiers in the chain (except for the first classifier) [26]. A drawback to CC is that the class variables' ordering is decided at random and this has effects on the classification rates [26], [27].

CCC consists of $q$ base binary classifiers (in our case the FSNB) linked circularly in a chain, creating a ring architecture (see Fig. 6). As in CC, each classifier at succeeding positions $2,3, \ldots, q$ aggregates as inputs the predicted classes of its previous classifiers. The circular configuration is generated 

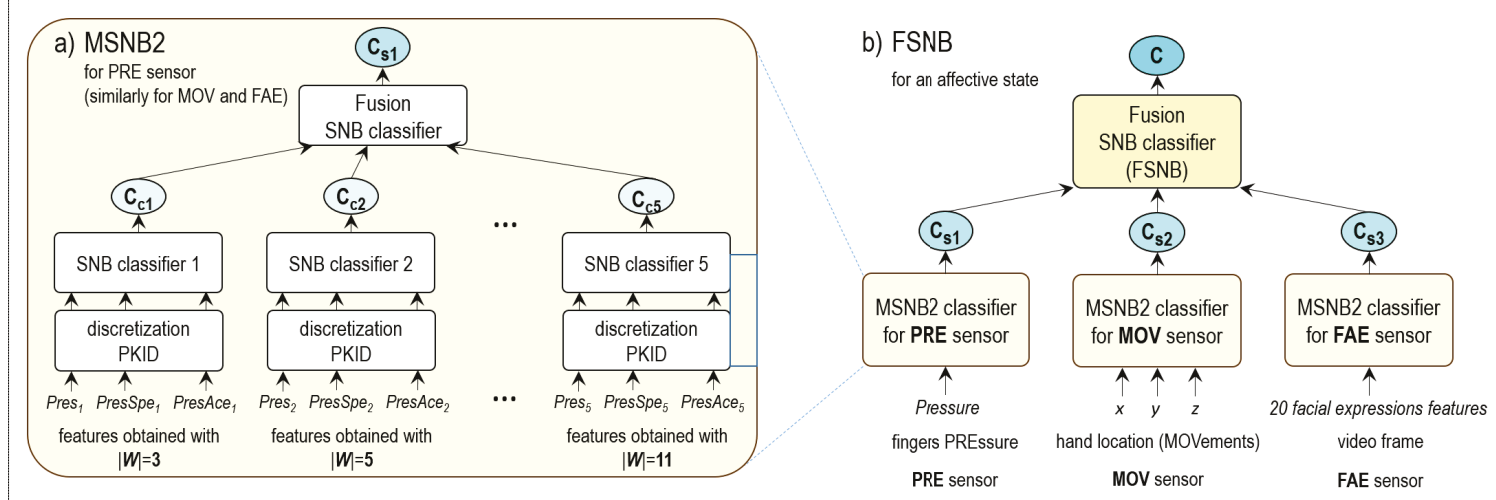

Fig. 5. Multiresolution Semi-Naive Bayesian (MSNB2) classifier and Late Fusion using SNBC (FSNB). a) MSNB2 is a binary classifier which combines a set of parallel windows $W$ of different odd sizes, $|W|=3,5,7,9,11$; all concurrently centred around the same frame of the respective sensor. PKID is a discretization method called Proportional k-interval discretization [25] to handle the numeric features. b) FSNB is a binary classifier which contains a MSNB2 classifier for each sensor (PRE, MOV, and FAE) and makes a late fusion using SNBC. FSNB is the multimodal affective states recognizer for an affective state. Acronyms meanings: $C, C_{c k}, C_{s j}=$ class of the respective classifier for the same affective state, e.g. anxiety; Pres $=$ pressure, Pres $S p e=$ pressure speed and PresAce $=$ pressure acceleration.

after the first "cycle" or iteration when the predicted classes of the classifiers at positions $2, \ldots, q$ are entered as additional features to the first one in the chain. The propagation of the predicted classes continues to the succeeding classifiers $(2,3, \ldots, q)$, and this mechanism is repeated for $N$ iterations or until convergence.

$\begin{gathered}\text { position 1 } \\ \text { affective state 1 }\end{gathered}$
$\begin{gathered}\text { position 2 } \\ \text { affective state 2 }\end{gathered}$ $\begin{gathered}\text { position 3 } \\ \text { affective state 3 }\end{gathered}$

Fig. 6. Circular Classifier Chains (CCC). At the first iteration, the predicted classes $C_{j}^{\prime}, j \in\{1,2, \ldots, q-1\}$ are propagated as classifier chains (CC). $\vec{A}$ is the feature vector (in our case, the feature vector of PRE, MOV, and FAE) Then, for the second iteration, the classifier at position 1 receives the predicted classes from the last classifier (the one at position $q$ ) and the other classifiers (positions $2,3, \ldots, q-1$ ). After that, the propagation process continues to the succeeding classifiers in the chain. The process is repeated until convergence or until CCC reached a maximum number of iterations.

\section{EXPERIMENTS AND RESULTS}

CCC models were independently trained for each patient to predict the occurrence of the four states (tiredness, anxiety, pain, and engagement) in the multi-label classification scheme. Therefore, we had 5 CCC models, one for each patient. Each CCC involved the development of 4 FSNB classifiers, one for each state of the corresponding patient. Similarly, BR and CC models were independently developed for each patient, so we had $5 \mathrm{BR}$ and $5 \mathrm{CC}$ models, with the corresponding 4 FSNB classifiers for each one. Therefore, the three classifiers BR, CC and CCC, were implemented using FSNB as the base classifier for all of them.
The performance of CCC was evaluated against BR and $\mathrm{CC}$ (used as baselines), using several metrics for multi-label classification [28]: Global accuracy $(G A c c)$, Mean accuracy $(M A c c)$, Multi-label accuracy $(M L A c c)$ and $F$-measure.

Internal validity of the $\mathrm{BR}, \mathrm{CC}$, and $\mathrm{CCC}$ models was established using the stratified ten-fold cross replication mechanism across all the rehabilitation sessions.

The class variables' ordering for CC, and initially for CCC, was defined considering the BR results of the area under the curve (AUC) of the class variables. They were sorted in decreasing order according to these AUC results, interpreting that the class variables with worse outcomes should be at the last positions so they could receive more information from the class variables of the preceding positions.

\section{A. Experiment: Performance comparison of BR, CC and CCC}

Table I summarizes the classification results, mean \pm std. deviation (across the 5 patients and across the 10 folds of the cross-validation), of BR, CC and CCC. CCC was run with 8 iterations, and the system converged at the third iteration for all the patients. The experiments were done with a laptop Intel Core i7-8750H CPU, 8th Gen. $2.20 \mathrm{GHz}, 16 \mathrm{~GB}$ RAM with an operating system Windows 64 bits. CCC using FSNB during training (with 10 fold cross validation) had an execution time of (mean \pm std. deviation) $124.09 \pm 30.46 \mathrm{sec}$. which corresponds to $2.07 \pm 0.51 \mathrm{~min}$. The number of data samples was (mean \pm std.deviation) $5625.00 \pm 2237.93$ across the five patients. The best results for each metric are highlighted in bold type. Results reveal that CCC outperformed BR and $\mathrm{CC}$ for all the metrics. Significant differences (Friedman test, $p<0.05$, with post hoc analysis with Wilcoxon signed-rank tests with Bonferroni correction, $p<0.017$ ) were obtained for CCC.

Table II shows average AUC results for each state across the five patients and across the ten folds. Average AUC results of CCC were significantly higher than the ones of BR and 
TABLE I

Performance COMPARISONS BETWEen BR, CC AND CCC (mean \pm std.deviation) (ACROSS THE 5 PATIENTS AND ACROSS THE 10 FOLDS OF THE CROSS-VALIDATION). CCC WAS RUN WITH 8 ITERATIONS, AND THE CONVERGENCE WAS ACHIEVED AT ITERATION 3 FOR ALL THE PATIENTS. THE BEST RESULTS FOR EACH METRIC ARE HIGHLIGHTED IN BOLD TYPE.

\begin{tabular}{lllll}
\hline Classifier & GAcc & MAcc & MLAcc & F-measure \\
\hline BR & $0.878 \pm 0.121$ & $0.963 \pm 0.037$ & $0.908 \pm 0.092$ & $0.918 \pm 0.084$ \\
CC & $0.910 \pm 0.092$ & $0.970 \pm 0.031$ & $0.930 \pm 0.072$ & $0.937 \pm 0.067$ \\
CCC & $\mathbf{0 . 9 4 1} \pm \mathbf{0 . 0 5 9} \ddagger$ & $\mathbf{0 . 9 7 7} \pm \mathbf{0 . 0 2 3} \ddagger$ & $\mathbf{0 . 9 5 4} \pm \mathbf{0 . 0 4 6} \ddagger$ & $\mathbf{0 . 9 5 8} \pm \mathbf{0 . 0 4 3} \ddagger$ \\
\hline$\ddagger$ means significant differences between CCC and CC, and between CCC and BR (Friedman test, \\
\multicolumn{2}{c}{$p<0.05$, post hoc analysis with Wilcoxon signed-rank tests with Bonferroni correction, } \\
$p<0.017)$.
\end{tabular}

CC (Friedman test, $p<0.05$, with post hoc analysis with Wilcoxon signed-rank tests with Bonferroni correction, $p<$ 0.017). The states average results of CCC in decreasing order are tiredness, anxiety, pain, and engagement.

TABLE II

Performance Comparisons BetWeen BR, CC AND CCC (mean $\pm s t d$.deviation) (ACROSS THE 5 PATIENTS AND ACROSS THE 10 FOLDS OF THE CROSS-VALIDATION) USING AUC FOR EACH STATE. CCC WAS RUN WITH 8 ITERATIONS. THE BEST RESULTS FOR EACH STATE ARE HIGHLIGHTED IN BOLD TYPE.

\begin{tabular}{|c|c|c|c|c|}
\hline Classifier & Tiredness & Anxiety & Pain & Engagement \\
\hline $\mathrm{BR}$ & $0.957 \pm 0.050$ & $0.952 \pm 0.053$ & $0.943 \pm 0.067$ & $0.932 \pm 0.076$ \\
\hline $\mathrm{CC}$ & $0.964 \pm 0.042$ & $0.965 \pm 0.039$ & $0.949 \pm 0.052$ & $0.943 \pm 0.066$ \\
\hline $\mathrm{CCC}$ & $\mathbf{0 . 9 7 5} \pm \mathbf{0 . 0 3 2} \ddagger$ & $\mathbf{0 . 9 7 2} \pm \mathbf{0 . 0 3 4} \ddagger$ & $\mathbf{0 . 9 6 5} \pm \mathbf{0 . 0 4 6} \ddagger$ & $\mathbf{0 . 9 5 6} \pm \mathbf{0 . 0 5 2} \ddagger$ \\
\hline
\end{tabular}

\section{B. Dependency relationships between the states}

Since late fusion in FSNB for predicting the classes of a certain state, $\mathrm{S}$, creates a SNB model which receives the predicted classes for the other states, in addition to the features of PRE, MOV, and FAE of $\mathrm{S}$, it is possible to analyze the conditional probability tables (CPTs) of the other states given state S. These CPTs were generated after the eight iterations of CCC. CPTs can provide us information about the relationships of the states that SNBC considers to assess the presence or the absence of the state under consideration. In Fig. 7, the CPTs of each state are depicted. The information is organized in blocks a), b), c), and d) corresponding to the CPTs of tiredness, anxiety, pain, and engagement, respectively. Each table entry represents mean \pm std. deviation across the five patients. From the analysis of the tables, we can detect the following relationships of the states for the five patients:

1) Tiredness: When tiredness is present, engagement is not, nor anxiety or pain.

2) Anxiety: When there is anxiety, there is no engagement, and there is no tiredness either. But when anxiety is not present, pain is not present either.

3) Pain: When there is pain, there is anxiety too; but there is neither engagement nor tiredness.
4) Engagement: When engagement is present, none of the other states (tiredness, anxiety, and pain) is present.

This evidence seems to establish that there is a mutual exclusion between engagement and all the other states and some co-occurrences between pain and anxiety for the five patients during their rehabilitation sessions.

We also created the CPTs of the states directly from the tags assigned by the psychiatrists, and the obtained table entries (mean \pm std.deviation) were almost the same as the ones generated by CCC using FSNB.
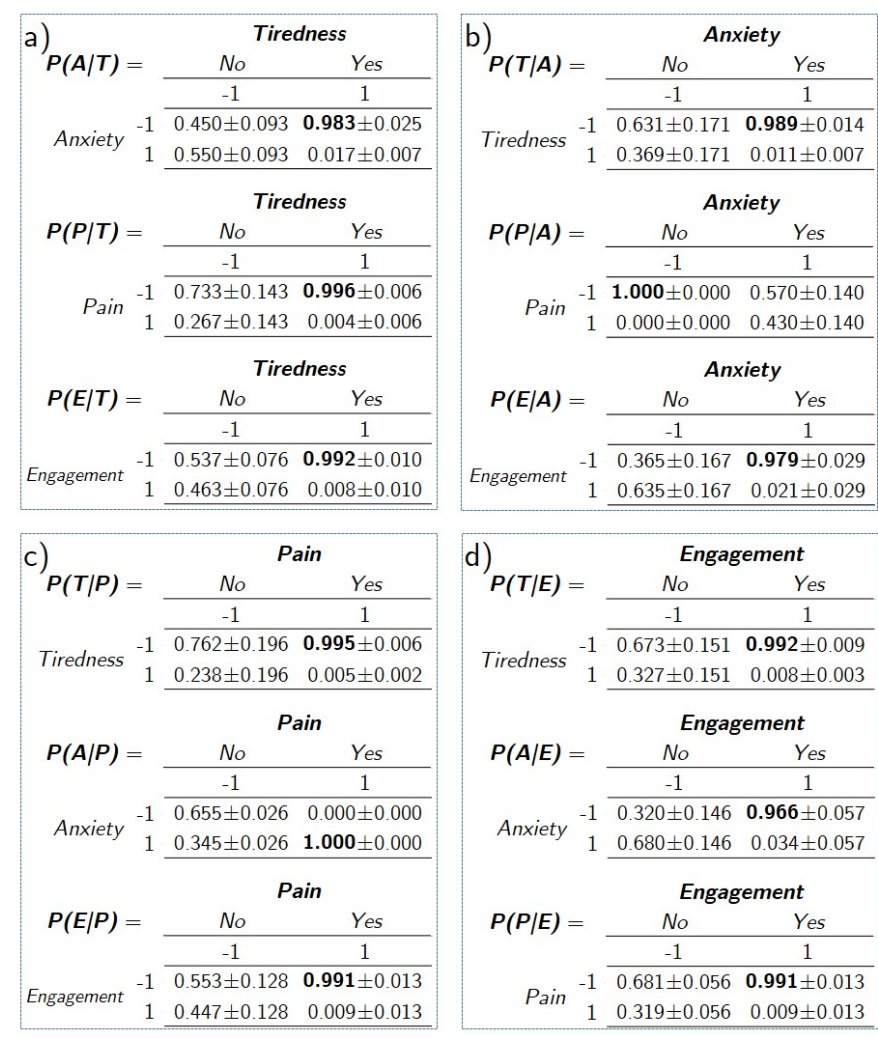

Fig. 7. Conditional Probability Tables (CPTs) generated by SNB models at the late fusion of FSNB, after the eight iterations of CCC. Each CPT entry represents mean $\pm s t d$. deviation across the five patients. The highest value for each CPT is highlighted in bold type. Part a) corresponds to tiredness CPTs, b) to anxiety CPTs, c) to pain CPTs, and d) to engagement CPTs.

\section{DISCUSSION}

As a consequence of the proposed computational model, CCC using FSNB as its base classifier, we have obtained a late fusion process in FSNB (for predicting the classes of an affective state) that not only considers the information from each modality (PRE, MOV, and FAE), but that also includes the correlation information with the other affective states into consideration. In this way, the fusion treats more that only the modalities involved in the problem. An additional contribution of CCC using FSNB as its base classifier, is that we can extract information about the mutual exclusion relationships and co-occurrences relationships between the states. This classification scheme has been beneficial for the 
prediction of the states in the dataset we used since all the patients' states were recognized with results over $94 \%$. So the proposed computational model is promising for affective states recognition. This model also has the advantage of simplicity, efficiency, and the consideration of dependency relationships between the states. A drawback is that the model does not consider the problem of a missing sensor.

\section{CONCLUSIONS AND FUTURE WORKS}

The relationships between tiredness, anxiety, pain, and engagement in the rehabilitation of some people after stroke have been studied using a multimodal computational model (FSNB) combined with the circular classifier chains (CCC). The computational model detected the relationships of mutual exclusion between engagement and all the other states and co-occurrences between pain and anxiety for the five patients during their rehabilitation sessions. Our proposal of $\mathrm{CCC}$ using FSNB as its base classifier outperformed CC and BR significantly (both $\mathrm{CC}$ and BR using FSNB as the base classifier too), with classification rates over $94 \%$. Additionally, the model converged after only 3 iterations for all the patients. Consequently, the proposed computational model is promising for its results, for its simplicity and because it maintains the $\mathrm{CC}$ efficiency.

As future work, the late fusion using SNBC can be replaced using other Bayesian classifiers. Additionally, the problem of a missing sensor will be addressed too. A larger trial is necessary to confirm whether this apparent trend can be generalized to the population considered.

\section{REFERENCES}

[1] L. E. Sucar, R. Leder, J. Hernández, I. Sánchez, and G. Azcárate, "Clinical evaluation of a low-cost alternative for stroke rehabilitation," in Proceedings of the 11th International Conference on Rehabilitation Robotics (ICORR 2009). IEEE, 2009, pp. 863-866.

[2] L. E. Sucar, F. Orihuela-Espina, R. L. Velazquez, D. J. Reinkensmeyer, R. Leder, and J. Hernández-Franco, "Gesture therapy: An upper limb virtual reality-based motor rehabilitation platform," IEEE Transactions on Neural Systems and Rehabilitation Engineering, vol. 22, no. 3, pp. 634-643, 2014.

[3] S. M. Ávila Sansores, "Adaptación en línea de una política de decisión utilizando aprendizaje por refuerzo y su aplicación en rehabilitación virtual," Master's thesis, Instituto Nacional de Astrofísica, Óptica y Electrónica (INAOE), Puebla, México., 2013.

[4] L. E. Sucar, S. M. Ávila-Sansores, and F. Orihuela-Espina, "User modelling for patient tailored virtual rehabilitation," in Foundations of Biomedical Knowledge Representation. Springer, 2015, pp. 259-278.

[5] K. Trohidis, G. Tsoumakas, G. Kalliris, and I. P. Vlahavas, "Multilabel classification of music into emotions." in Proceedings of the 9th International Conference on Music Information Retrieval (ISMIR), vol. 8, 2008, pp. 325-330.

[6] T. Olugbade, A. Williams, and N. Bianchi-Berthouze, "The relationship between guarding, pain, and emotion." PAIN Report, vol. In press, 2019.

[7] S. Wang, Z. Wang, and Q. Ji, "Multiple emotional tagging of multimedia data by exploiting dependencies among emotions," Multimedia Tools and Applications, vol. 74, no. 6, pp. 1863-1883, 2015.

[8] S. Wang, J. Wang, Z. Wang, and Q. Ji, "Multiple emotion tagging for multimedia data by exploiting high-order dependencies among emotions," IEEE Transactions on Multimedia, vol. 17, no. 12, pp. 21852197, 2015.

[9] M. J. Pazzani, "Searching for dependencies in Bayesian classifiers," in Learning from Data. Springer, 1996, pp. 239-248.

[10] L. E. Sucar, Probabilistic Graphical Models: Principles and Applications. Springer, 2015.
[11] J. J. Rivas, F. Orihuela-Espina, L. Palafox, N. Berthouze, M. del Carmen Lara, J. Hernández-Franco, and E. Sucar, "Unobtrusive inference of affective states in virtual rehabilitation from upper limb motions: A feasibility study," IEEE Transactions on Affective Computing, 2018.

[12] J. J. Rivas, F. Orihuela-Espina, and L. E. Succar, "Circular chain classifiers," in Proceedings of Machine Learning Research. 9th International Conference on Probabilistic Graphical Models, vol. 72, 2018, pp. 380391.

[13] J. J. Rivas, F. Orihuela-Espina, and L. E. Sucar, "Recognition of affective states in virtual rehabilitation using late fusion with SemiNaive Bayesian classifier," in Proceedings of the 13th EAI International Conference on Pervasive Computing Technologies for Healthcare (PervasiveHealth 2019). ACM, 2019, pp. 308-313.

[14] P. Philippot, "Inducing and assessing differentiated emotion-feeling states in the laboratory," Cognition and emotion, vol. 7, no. 2, pp. 171193, 1993.

[15] J. J. Gross and R. W. Levenson, "Emotion elicitation using films," Cognition \& emotion, vol. 9, no. 1, pp. 87-108, 1995.

[16] T. Olugbade, A. Singh, N. Bianchi-Berthouze, N. Marquardt, M. Aung, and A. Williams, "How can affect be detected and represented in technological support for physical rehabilitation?" ACM Transactions on Computer-Human Interaction (TOCHI), vol. 26, no. 1, 2019.

[17] Z. Wang, S. Wang, M. He, Z. Liu, and Q. Ji, "Emotional tagging of videos by exploring multiple emotions' coexistence," in Proceedings of the 10th IEEE International Conference and Workshops on Automatic Face and Gesture Recognition (FG 2013). IEEE, 2013, pp. 1-6.

[18] A. Bandini, S. Orlandi, H. J. Escalante, F. Giovannelli, M. Cincotta, C. A. Reyes-Garcia, P. Vanni, G. Zaccara, and C. Manfredi, "Analysis of facial expressions in parkinson's disease through video-based automatic methods," Journal of neuroscience methods, vol. 281, pp. 7-20, 2017.

[19] M. Soleymani, J. Lichtenauer, T. Pun, and M. Pantic, "A multimodal database for affect recognition and implicit tagging," IEEE Transactions on Affective Computing, vol. 3, no. 1, pp. 42-55, 2012.

[20] R. O. Duda, P. E. Hart et al., Pattern classification and scene analysis. Wiley New York, 1973, vol. 3.

[21] M. Martínez-Arroyo and L. E. Sucar, "Learning an optimal naive Bayes classifier," in Proceedings of the 18th International Conference on Pattern Recognition, 2006. (ICPR 2006), vol. 3. IEEE, 2006, pp. 12361239.

[22] M. Martínez Arrollo, "Aprendizaje de clasificadores bayesianos estáticos y dinámicos," Ph.D. dissertation, Instituto Tecnológico y de Estudios Superiores de Monterrey, México, 2007.

[23] C. Chow and C. Liu, "Approximating discrete probability distributions with dependence trees," IEEE Transactions on Information Theory, vol. 14 , no. 3 , pp. $462-467,1968$.

[24] J. J. Rivas, "Clasificador Semi-Naïve Bayes con multiresolución para la estimación de estados afectivos: Aplicación en rehabilitación virtual," Master's thesis, Instituto Nacional de Astrofísica, Óptica y Electrónica (INAOE), Puebla, México., 2015.

[25] Y. Yang and G. I. Webb, "Proportional k-interval discretization for naivebayes classifiers," in Proceedings of the 12th European Conference on Machine Learning (ECML 2001). Springer, 2001, pp. 564-575.

[26] J. Read, B. Pfahringer, G. Holmes, and E. Frank, "Classifier chains for multi-label classification," in Proceedings of the Joint European Conference on Machine Learning and Knowledge Discovery in Databases. Springer, 2009, pp. 254-269.

[27] E. C. Goncalves, A. Plastino, and A. A. Freitas, "A genetic algorithm for optimizing the label ordering in multi-label classifier chains," in Proceedings of the 25th International Conference on Tools with Artificial Intelligence (ICTAI). IEEE, 2013, pp. 469-476.

[28] C. Bielza, G. Li, and P. Larrañaga, "Multi-dimensional classification with Bayesian networks," International Journal of Approximate Reasoning, vol. 52, no. 6, pp. 705-727, 2011. 